\title{
Sobre la relación entre liberalización y eficiencia productiva en el sector ferroviario en Europa
}

\author{
CARlos Lérida NAVARro a , José MANUEl TránChez MARTín a \\ a Universidad Nacional de Educación a Distancia (UNED), Facultad de Derecho, c/ Obispo Trejo, 2, \\ 28040 Madrid,España.E-mail: carlos.lerida@madrimasd.net, jtranchez@cee.uned.es
}

\begin{abstract}
RESUMEN
El objetivo de este artículo es contrastar si las políticas de liberalización del sector ferroviario, promovidas desde la Comisión Europea para incrementar el peso relativo del transporte ferroviario respecto a otros modos de transporte, han tenido efecto en la eficiencia productiva de los sistemas ferroviarios europeos que las han adoptado. Para ello se aporta como novedad la utilización de una medida del grado de liberalización de los sistemas ferroviarios europeos y su puesta en relación con los índices de eficiencia de dichos sistemas calculados según la metodología DEA por programas. Los resultados obtenidos, cuando se analizan los datos agregados, aportan evidencia empírica de una asociación positiva entre la eficiencia y la liberalización de los sistemas ferroviarios. Cuando se realizan análisis individualizados por países esta relación se difumina, de manera que no es posible destacar un grado de liberalización óptimo o un modelo de gestión que pueda servir de referencia.
\end{abstract}

Palabras clave: Liberalización, eficiencia, transporte ferroviario.

\section{On the Relation between Deregulation and Productive Efficiency in European Railway Sector}

\begin{abstract}
Among the European policies focused on increasing the railways market share versus other transportation modals, deregulation policies are the most relevant. The aim of this article is to contrast if these deregulation policies developed in European railways systems are working from the efficiency perspective. The main contribution of the paper is the use of a liberalization index that is related with efficiency indexes estimated by Programmatic Efficiency methodology. Results using aggregated data show empirical evidence of a positive association between deregulation and efficiency. However results analyzing each individual railway system do not show an evident relationship, so it is not possible to set an optimal deregulation level or even a management model that can be used as a reference in efficiency terms.
\end{abstract}

Keywords: Deregulation, Efficiency, Railways.

Clasificación JEL: L51, L92, C14, D24, H44

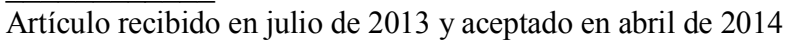

Artículo disponible en versión electrónica en la página www.revista-eea.net, ref. ə-32208 


\section{INTRODUCCIÓN}

La organización del transporte ferroviario ha estado durante décadas sometida al máximo grado de control público a través de un modelo de monopolio. Desde sus comienzos, al tratarse de un sector con importantes economías de escala, se generó de forma natural una tendencia hacia la concentración de empresas ferroviarias. El paso hacia un modelo de monopolio público se produjo en muchos países europeos al finalizar la Segunda Guerra Mundial, cuando hubo que nacionalizar estas grandes compañías previamente existentes debido a sus problemas financieros. Todo ello derivó en un modelo en el que los sistemas ferroviarios se organizaron bajo una única empresa pública, proveedora simultáneamente de los servicios de transporte de viajeros y mercancías y de la infraestructura necesaria para el funcionamiento de este modo.

El modelo anteriormente indicado se halla actualmente en revisión y está atravesando por un proceso simultáneo de liberalización y privatización. De este modo, se están ensayando diferentes opciones para introducir un mayor grado de competencia en el mercado, así como para permitir la incorporación de operadores ferroviarios privados que disputen el monopolio al sector público.

La Comisión de la Unión Europea, con el objetivo de revitalizar y fomentar el tráfico ferroviario frente a otros modos de transporte, presentó en 1990 la comunicación, sin carácter legislativo, titulada "Política Ferroviaria Comunitaria". Las propuestas de esta comunicación fueron desarrolladas en varias Directivas posteriores que se agrupan en los denominados "Paquetes Ferroviarios".

El "Primer Paquete Ferroviario" (Directivas 2001/12, 2001/13 y 2001/1), conocido como "de infraestructura", fue diseñado para, asegurar la independencia de los gestores de infraestructuras estableciendo un régimen normativo transparente para la asignación de las capacidades y las tarifas de acceso a la infraestructura ferroviaria en toda la UE. En 2004 se adopta el denominado "Segundo Paquete Ferroviario" (Directivas 2004/49, 2004/50 y 2004/51 y Reglamento 881/2004), con los objetivos de apertura del mercado del transporte ferroviario y la creación de una Agencia Ferroviaria Europea encargada de garantizar la seguridad e interoperabilidad. El "Tercer Paquete Ferroviario" aprobado en 2007 (Directivas 2007/58 y 2007/59 y Reglamento 1371/2007) tuvo como objetivo promover un mercado interior de servicios ferroviarios amparado en la competencia y la protección de los derechos de los viajeros. Por último, el 30 de enero de 2013 se ha presentado el borrador de un "Cuarto Paquete Ferroviario" que comprende seis propuestas legislativas destinadas a reforzar el papel de los gestores de la infraestructura, que controlarán todas las funciones esenciales relacionadas con la explotación de la red, incluida la planificación de la inversión en infraestructuras, la explotación diaria, el mantenimiento, así como el establecimiento de horarios. 
Todas estas Directivas se han ido incorporando a la legislación de los diferentes países comunitarios con diferentes ritmos e intensidades. La particular aplicación de esta normativa en cada país ha cristalizado en diferentes modelos de gestión del sector ferroviario, que representan diferentes niveles de competencia y liberalización y que generan resultados variados en términos de eficiencia productiva. Con objeto de tener una información sintética, en la Tabla 1, se presenta una clasificación propia de los principales modelos de gestión del sistema ferroviario atendiendo a dos de sus elementos más destacados: por un lado, el nivel de separación existente entre las actividades de dotación de la infraestructura (gestor) y prestación del servicio (operador) y, por otro lado, el nivel de competencia efectiva en función del número de operadores diferentes que realizan tráficos en el sistema. La información necesaria para clasificar los sistemas según el primer criterio (separación gestor-operador) ha sido obtenida de la revisión de las legislaciones respectivas. La información para el segundo criterio (nivel de competencia) ha sido obtenida a partir del "Índice de liberalización" ofrecido por la Universidad Humbolt, (Kirchner 2002, 2004, 2007 y 2011) que se introduce en el apartado 4.

Tabla 1

Diferentes modelos de organización del sector ferroviario en Europa

\begin{tabular}{|l|c|c|}
\hline \multicolumn{2}{|c|}{$\begin{array}{c}\text { Nivel de separación } \\
\text { gestor - operador }\end{array}$} & \multicolumn{2}{c|}{ Nivel de competencia real en operadores } \\
\cline { 2 - 3 } Integración vertical pública & Nulo o escaso & Amplio \\
\hline Integración vertical privada & $\begin{array}{c}\text { Lituania, Luxemburgo, } \\
\text { Irlanda }\end{array}$ & $\begin{array}{c}\text { Alemania, Austria, Italia, } \\
\text { Polonia, Suiza, Bélgica, } \\
\text { Letonia. Eslovenia, Hungria }\end{array}$ \\
\hline Separación infraestructura en gestor público & $\begin{array}{c}\text { Francia España, Portugal, } \\
\text { Grecia, Finlandia, } \\
\text { Eslovaquia }\end{array}$ & $\begin{array}{c}\text { Suecia, Holanda, Dinamarca, } \\
\text { Chequia, Noruega, Rumanía, } \\
\text { Bulgaria }\end{array}$ \\
\hline Separación infraestructura en gestor privado & - & Reino Unido \\
\hline
\end{tabular}

Fuente: Elaboración propia.

La evidencia empírica de trabajos anteriores que analizan los efectos del proceso de liberalización sobre la eficiencia en el sector del transporte ferroviario no es concluyente. Entre otros Friebel, Ivaldi y Vibes (2003), Wetzel (2008) y Cantos, Pastor y Serrano (2010) muestran como algunas medidas liberalizadoras concretas afectan, en algunos casos, positivamente a la eficiencia, sin que se obtengan conclusiones del proceso liberalizador en su conjunto. La justificación del presente trabajo se fundamenta en aportar evidencia empírica adicional sobre los efectos que los diferentes grados de liberalización adoptados puedan tener en los sistemas de transporte ferroviarios estudiados.

La estructura del trabajo es la siguiente. Tras este primer apartado introductorio, en el apartado segundo se plantean los objetivos del trabajo. El tercer 
apartado expone la metodología seguida en el estudio, justificando la elección del modelo de estimación empleado, DEA (Data Envelopment Analysis). La descripción de las variables utilizadas y los datos disponibles para cada una de ellas se muestran en el apartado cuarto. La presentación de los resultados obtenidos y la extracción de conclusiones respecto a los mismos se llevan a cabo en los apartados quinto y sexto respectivamente.

\section{OBJETIVOS}

El objetivo del trabajo es obtener evidencia empírica de la posible relación existente entre grado de liberalización del sector ferroviario y nivel de eficiencia productiva alcanzada en el funcionamiento del mismo.

Dicho objetivo se estructura en dos fases. En la primera de ellas se evalúa la eficiencia técnica de los principales 27 sistemas ferroviarios europeos, entendida ésta como relación entre cantidades de inputs y outputs, a través de un modelo DEA. Esta evaluación se realiza en dos planos. Por un lado, en un plano individual, donde se estima el grado de eficiencia técnica de cada sistema ferroviario con relación a la frontera de sistemas ferroviarios más eficientes. Por otro lado, en un plano agregado, donde se procede a la construcción de dos escenarios agrupando sistemas ferroviarios basándose en el grado de liberalización alcanzado y se procede a la comparación entre los mismos.

En la segunda fase se incorporan los datos de "Índices de liberalización" obtenidos a partir de Kirchner (2002, 2004, 2007 y 2011) al efecto de obtener evidencia empírica de la relación entre la variable eficiencia calculada en la fase anterior y la variable liberalización. El estudio de la relación entre dichas variables se realizará a través de diversas herramientas: descriptiva, mediante la evaluación comparada de las medias de los subgrupos ineficientes en cada escenario con las medias del conjunto de sistemas de cada escenario y mediante el contraste del estadístico Kruskal-Wallis.

Este trabajo presenta varias innovaciones de interés respecto a trabajos anteriores sobre el tema. En primer lugar, la medición realizada de la eficiencia de cada sistema ferroviario, entendido como un todo y no centrándose solamente en medir la eficiencia técnica de las empresas operadoras. Por otro lado la utilización, para la comparación con los índices de eficiencia, de una medida agregada del grado de liberalización alcanzado por cada uno de los sistemas ferroviarios europeos que no era aplicada en los trabajos previos. Finalmente, la introducción para este sector de la metodología de eficiencia por programas que permite profundizar en la relación existente entre la variable liberalización y las variables utilizadas para el cálculo de la eficiencia, a partir de la identificación de diferencias de rendimiento entre los grupos que han implementado programas de liberalización y los que no. 
Adicionalmente, se presentan otros elementos metodológicos no aplicados en trabajos anteriores sobre la eficiencia de las empresas ferroviarias. Así, se introduce en la estimación un contraste previo de la homogeneidad de la muestra ${ }^{1}$ sobre la que se aplica la metodología DEA a partir de un "análisis de conglomerados jerárquicos". También se introduce la utilización del estadístico Kruskal-Wallis como herramienta para contrastar la existencia de evidencia empírica suficiente que permita aceptar la relación entre la variable eficiencia calculada y la variable liberalización, medida sobre la base de los "Índices de liberalización” obtenidos a partir de Kirchner (2002, 2004, 2007 y 2011).

\section{METODOLOGÍA}

La obtención de indicadores del grado de eficiencia técnica alcanzado por los sistemas ferroviarios, tanto de forma individual como de forma agregada, se hace utilizando la metodología DEA (Data Envelopment Analysis). Dicha metodología se aplica tanto en su modalidad de una única etapa, como en su variante de dos etapas, propuesta en Charnes, et al. (1981) y conocida como "eficiencia por programas".

Su utilización parece adecuada en este trabajo por diversas razones. En primer lugar, por ser una técnica útil en contextos multiproducto, como es el caso de los sistemas ferroviarios en los que se producen dos tipos de servicios, transporte de pasajeros y de mercancías. En segundo lugar, dado su carácter de técnica no paramétrica, evitando los errores que se pudieran cometer en la determinación de una función de producción previa. En tercer lugar, por su recomendable interés en sectores con influencia relevante del sector público. Finalmente, porque esta misma técnica ha sido utilizada convenientemente en muchos otros estudios del sector, entre los que cabe señalar a Oum y Yu (1994); Cowie (1999); o Cantos, Pastor y Serrano (2000).

La metodología DEA, utilizando la información de las cantidades empleadas de inputs y producidas de outputs por los sistemas ferroviarios analizados, permite optimizar una medida de eficiencia, estimando una frontera de producción eficiente y factible de forma empírica y, simultáneamente, evaluando la eficiencia de cada unidad observada medida como distancia a la frontera.

El modelo utilizado puede formalizarse en su versión dual como:

$$
\operatorname{Max}_{\theta, S+, S D-, \lambda} \theta_{0}+\varepsilon\left(\left(S_{D}-/ \sigma_{D}\right)+(S+/ \sigma)\right)
$$

sujeto a:

\footnotetext{
${ }^{1}$ Para un estudio de los requisitos de la homogeneidad de la muestra que exige esta técnica a las unidades evaluadas puede consultarse Golany y Roll (1989).
} 


$$
\begin{aligned}
& X \lambda+S_{D}=X_{0} \\
& Y \lambda-S=\theta_{0} Y_{0} \\
& S_{D}, S, \lambda>0
\end{aligned}
$$

Siendo:

$\mathrm{X}$ : matriz de inputs.

$\mathrm{Y}$ : matriz de outputs.

$\lambda$ : vector de parámetros solución del modelo.

$\mathrm{S}$ : variables de holgura solución del modelo.

$\sigma:$ desviaciones típicas.

$\theta_{0}$ : ratio de eficiencia del sistema ferroviario evaluado solución del modelo, definido:

$$
\begin{aligned}
& \theta_{0}=\frac{\sum U_{r} Y_{r k}}{\sum V_{i} X_{i k X}} \\
& U_{r}, V_{i}>0
\end{aligned}
$$

Siendo:

Yrk: el output r-ésimo del sistema ferroviario k-ésimo.

Xik: el intput i-ésimo del sistema ferroviario k-ésimo.

Ur: la ponderación del output r-ésimo.

Vi: la ponderación del input i-ésimo.

Para el cálculo de los niveles de inputs y outputs que cada entidad debería tener para ser eficiente (Target Values o Valores Objetivo) basta con aplicar:

$X \lambda=X_{0}-S_{D}$ : Sustraer la variable de holgura al valor real.

$Y \lambda=\theta_{0} Y_{0}+S:$ Valor real por ratio de eficiencia más variable de holgura.

Dentro de las posibilidades que ofrece la metodología DEA, en el presente estudio se adopta un modelo DEA dual, CCR (rendimientos constantes a escala) y con orientación al input.

En la mayor parte de las aplicaciones DEA el modelo empleado en la evaluación de la eficiencia es el programa lineal DEA-CCR dual (en forma envolvente), preferido al programa lineal DEA-CCR primal (en forma multiplicativa). Siendo $\mathrm{n}$ el número unidades de decisión de la muestra, $\mathrm{s}$ el número de outputs diferentes y $\mathrm{m}$ el número de inputs distintos, el modelo dual impone $\mathrm{s}+\mathrm{m}$ restricciones frente a las $\mathrm{n}+1$ restricciones del programa lineal primal. Por tanto, como el número de unidades con las que se trabaja suele ser mucho mayor que la suma del número de inputs y outputs se prefiere el modelo dual para ser resuelto (Coelli, et al., 1998).

El modelo DEA-CCR (rendimientos constantes a escala) proporciona una 
medida de "eficiencia técnica global", mientras que el modelo DEA-BCC ${ }^{2}$ (rendimientos variables a escala) estima una medida de "eficiencia técnica pura" neta de cualquier efecto de escala. El modelo CCR es preferido al BCC en los análisis de eficiencia del sector ferroviario llevado a cabo por Oum y Yu (1994) ${ }^{3}$, Cantos, Pastor y Serrano (2000), Hilmola (2006) y Asmild, et al. (2009) ${ }^{4}$ Así, en línea con Cantos, Pastor y Serrano (2000) el modelo DEA que asume CCR presenta resultados de eficiencia iguales o mayores que los que arroja con BCC, luego la opción de rendimientos constantes a escala es una hipótesis más exigente con la eficiencia que la de rendimientos variables. También Esteban y Coll, (2003) destacan que la frontera sobre la que se proyecta el cálculo de eficiencias en el modelo DEA-CCR produce un menor número de entidades eficientes y puntuaciones de eficiencia menores entre todos los sistemas analizados, lo que conlleva mayor exigencia. El cálculo de la eficiencia en el presente trabajo se realiza con la finalidad de comparar la asociación entre esta variable y el grado de liberalización, por lo que una medida de la eficiencia que recoja su valor global frente a uno de sus componentes únicamente, parece más adecuada, sin que sea relevante para este propósito aislar el efecto de las economías de escala. Finalmente, bajo el supuesto de rendimientos constantes a escala, las medidas de eficiencia técnica input y output orientadas coinciden, medidas que no son necesariamente iguales en el caso de rendimientos variables.

Respecto a la orientación al input, la industria del transporte ferroviario está asociada normalmente con infraestructuras que generan costes hundidos, de manera que una vez que la red ferroviaria es construida, su output es aproximadamente fijo dentro de algún rango. En este caso, la cuestión de cómo utilizar eficientemente los inputs existentes es la clave para ahorrar costes en la producción de servicios de transporte ferroviario. Adicionalmente, según Farrel (1957), si se analiza un caso con varios inputs y un solo output lo más adecuado sería elegir una medida orientada al output ya que este sería un escalar y no un vector. Por tanto, a sensu contrario de la recomendación de Farrel, en nuestro caso en los que existen dos outputs parece acertada la teórica orientación hacia

\footnotetext{
${ }^{2}$ Banker, et al. (1989) relajan este supuesto al permitir que la tecnología presente rendimientos variables a escala. La operatividad de este supuesto se traduce en la adición de la denominada restricción de convexidad $\lambda=1$ en el modelo CCR, eliminando de esta forma la influencia de la escala de producción.

${ }^{3}$ Por permitir el cálculo de toda la efíciencia observable a partir de los datos input/output y la comparación con otros métodos no paramétricos de cálculo de la eficiencia como la Productividad Total de los Factores.

${ }^{4}$ En concreto, Asmild, et al. (2009) aplican una metodología Multi-directional Efficiency Analysis (MEA) asumiendo rendimientos de escala constantes y una orientación al input.
} 
los inputs ${ }^{5}$ en este caso, dado que además, en la práctica, los indicadores de eficiencia bajo el modelo CCR coinciden para ambas orientaciones.

Adicionalmente a la estimación DEA en una etapa se utiliza el cálculo de la "eficiencia por programas" (programmatic o interprogram efficiency) o por etapas con una doble finalidad. En primer lugar, identificar diferencias de rendimiento entre los grupos que han implementado programas de liberalización y los que no, contrastando así los resultados obtenidos con la técnica DEA en una sola etapa y, de este modo, comprobando la robustez del modelo propuesto. En segundo lugar, profundizar en la relación existente entre la variable liberalización y las variables estudiadas para el cálculo de la eficiencia, a partir de las holguras que se estiman en la segunda etapa.

Esta metodología por etapas tiene como objetivo estimar la eficiencia debida a la variable de entorno, en nuestro caso la implementación de las políticas de liberalización de los sistemas ferroviarios. En una primera etapa, se divide la muestra en dos submuestras, correspondientes a las categorías proporcionadas por la variable de entorno (sistemas ferroviarios más y menos liberalizados), y se estima separadamente la frontera mediante DEA, obteniendo así los niveles de eficiencia de gestión (managerial o intra-program efficiency). A continuación, para aquellos sistemas que se hayan mostrado ineficientes se sustituyen los valores observados de input u output por sus valores objetivos (proyectados sobre la frontera), cada uno en su submuestra correspondiente, eliminando así las ineficiencias de gestión detectadas. De este modo, la holgura residual contendría dos componentes: el efecto del entorno (políticas de liberalización) y el del error. Así se elimina de cada submuestra la ineficiencia intraprograma de las unidades ineficientes.

En la segunda etapa se estima la frontera y el índice de eficiencia con toda la muestra y con los datos corregidos. El nuevo índice de eficiencia por programas recoge el efecto causado únicamente por diferencias entre las categorías de la variable de entorno (implementación o no de las políticas de liberalización), de tal forma que las holguras resultantes proporcionan la información requerida para los contrastes estadísticos que permiten estudiar dicho efecto.

En otro orden de cosas, en los estudios de eficiencia tienen ventaja aquellos trabajos que concentran su atención en pequeñas áreas, con un grupo reducido de unidades y que requieren del uso de pocas variables. Por ello, en nuestro caso, es conveniente delimitar el estudio a sistemas ferroviarios lo suficientemente homogéneos para que no queden incluidas diferencias significativas entre

\footnotetext{
${ }^{5}$ La orientación al input se emplea cuando se calcula el modelo BCC en el estudio de análisis de eficiencia de Dios, et al. (2006) para la industria conservera murciana y en Guarnido, et al. (2007), a efectos de comparar con el modelo CCR, para otro sector en red, las telecomunicaciones.
} 
sistemas que interfieran el análisis de eficiencia ${ }^{6}$. Para ello se propone la utilización de la técnica de análisis de conglomerados jerárquico. Este análisis permite evidenciar la existencia o no de diferentes grupos de sistemas ferroviarios con características propias. En el caso de existir muchos grupos y/o ser estos muy numerosos lo recomendable será configurar diferentes modelos de DEA, de tal forma que, se puedan comparar en ellos sistemas ferroviarios que guarden homogeneidad en las variables estudiadas. De no ser así, podrían incorporarse en un único modelo los sistemas ferroviarios, como unidades de decisión del modelo DEA, sin riesgo a obtener resultados sesgados por falta de homogeneidad de los sistemas estudiados.

Para el desarrollo de este ejercicio se ha optado por el "método de vinculación inter-grupos", dado que aprovecha la información de todos los miembros de los dos conglomerados que se comparan. Se cuantifica la proximidad entre dos grupos calculando la media de las distancias entre objetos de ambos grupos o la media de las similitudes entre objetos de ambos grupos. La distancia entre los conglomerados $\mathrm{r}$ y s (dRS) se calcula como la distancia promedio existente entre todos los pares de elementos de ambos conglomerados y vendría dada por:

$$
d_{r s}=\frac{1}{n_{r} n_{s}} \sum_{j \in r} \sum_{k \in s} d(j, k)
$$

donde $\mathrm{d}(\mathrm{j}, \mathrm{k})$ es la distancia entre los objetos $\mathrm{j}$ y $\mathrm{k} \mathrm{y} \mathrm{n}_{\mathrm{r}}, \mathrm{n}_{\mathrm{s}}$ son los tamaños de los grupos $\mathrm{r}$ y s, respectivamente.

De la aplicación preliminar de la técnica de conglomerados jerárquicos se desprende que la homogeneidad es predominante entre los valores que presentan las variables utilizadas en el posterior análisis DEA de los sistemas ferroviarios y por tanto procede la aplicación de esta técnica. Para un análisis detallado de los resultados del ejercicio de conglomerados jerárquicos con dos variantes, se puede examinar el Anexo 1 de este trabajo.

Finalmente, otra novedad metodológica a los efectos de contrastar la hipótesis estudiada de asociación entre liberalización y eficiencia es la utilización del estadístico Kruskal-Wallis. Esta prueba tiene por objeto el análisis de la varianza por rangos para contrastar si las muestras comparadas, no relacionadas, se originan de una misma población. Cuando el test genera resultados significativos al menos una de las muestras es diferente respecto al resto, sin identificar dónde se encuentran, ni cuántas son, estas diferencias.

Suponiendo que las medias/medianas de los grupos son iguales, la variación entre grupos (intergrupal) es comparable a la variación entre individuos (intra-

\footnotetext{
${ }^{6}$ Estas entidades deben ser unidades comparables, en el sentido que todas las entidades consumen los mismos inputs, en diferentes cantidades, para producir el mismo conjunto de outputs, en distintas cantidades, Pastor (2000).
} 
grupal). Si la primera es muy superior a la segunda, puede indicar que las medias/medianas en realidad no son iguales.

El objeto del contraste por tanto es:

Hipótesis nula $\left(\mathrm{H}_{0}\right)$ : las $\mathrm{k}$ medianas son todas iguales, siendo $\mathrm{k}$ el número de valores distintos de la variable explicada.

Hipótesis alternativa $\left(\mathrm{H}_{1}\right)$ : al menos una de las medianas es diferente.

El estadístico de contraste K-W se calcula como

$$
K-W=\frac{\frac{12}{n(n+1)} \sum_{m=1}^{r} \frac{1}{n_{m}}\left[R_{m}-E\left[R_{m}\right]\right]^{2}}{1-\frac{\sum_{j=1}^{k}\left(d_{j}^{3}-d_{j}\right)}{n^{3}-n}}
$$

Siendo,

$r$ : el número de grupos.

$\mathrm{R}_{\mathrm{m}}$ : la suma de rangos de cada grupo $\mathrm{m}$.

$\mathrm{E}\left[\mathrm{R}_{\mathrm{m}}\right]$ : el valor medio de los rangos.

$\mathrm{d}_{\mathrm{j}}$ : el número de empates en $\mathrm{j}=1, \ldots, \mathrm{k}$.

Este estadístico sigue una distribución Chi-Cuadrado con $\mathrm{r}-1$ grados de libertad.

\section{DATOS Y VARIABLES}

Los datos utilizados para medir la eficiencia de los sistemas ferroviarios analizados se han obtenido de la Union Internationale des Chemin de Fer (UIC) que cuenta con información sobre las variables necesarias para realizar estimaciones siguiendo la técnica DEA (inputs y outputs) para todos los países de nuestro estudio entre 1991 y $2011^{7}$.

Las variables utilizadas en el modelo son las habitualmente utilizadas en la literatura sobre la eficiencia en el transporte ferroviario. Se incorporan como inputs del modelo el factor productivo capital (que comprendería las locomotoras [LOC] y la infraestructura sobre la que se opera la red [LON]) y el factor trabajo [PER]. Adicionalmente, dado que la realización de trayectos tanto de pasajeros como de mercancías que se producen en un sistema ferroviario requiere de la existencia de población y de su distribución en el territorio, se incorpora la densidad de población en el territorio [DEN] como input del

\footnotetext{
${ }^{7}$ UIC (2011) Railways time-series data 1991-2011.
} 
modelo ${ }^{8}$. Respecto a los outputs, es generalmente aceptado en la literatura que la medición del output del servicio de transporte a partir de las variables toneladas-kilómetro $[\mathrm{TKM}]$ y pasajeros-kilómetro [PKM] es más precisa que a través de las cantidades nominales de toneladas y/o pasajeros transportados, dado que en este caso no se tienen en cuenta la longitud de los trayectos realizados. Un repaso interesante a las variables input y output incorporadas en diferentes estudios sobre la eficiencia del transporte ferroviario se puede consultar en Affuso, et al. $(2002)^{9}$.

La medida del grado de liberalización de los diferentes sistemas ferroviarios se ha obtenido a partir del índice elaborado en la Universidad Humboldt de Berlín por Kirchner (2002, 2004, 2007 y 2011). El índice de liberalización [LIB Index] se calcula a partir de 230 variables medidas para cada país. Se conforma como la suma ponderada de un subíndice que cuantifica el grado de liberalización plasmado en la regulación aplicable y que pondera un 20\% [LEX Index], y de un subíndice que mide las condiciones de apertura que se presentan en la práctica y que pondera al 80\% [ACCESS Index]. Para el cálculo del LEX Index se tienen en cuenta aspectos como la regulación del acceso al mercado (45\% del subíndice), la existencia y competencias de una autoridad reguladora (30\%) y la estructura organizativa del operador nacional heredero del monopolio público (25\%). El valor del ACCESS Index depende de las barreras de tipo operativo (50\% de ponderación), la accesibilidad del mercado (25\%), las barreras administrativas (20\%) y las barreras informativas (5\%). En general, resulta un mayor grado de liberalización normativa ( $L E X$ Index) que lo que reflejan en la práctica las condiciones de acceso para operadores externos (ACCESS Index).

La Tabla 2 presenta las variables utilizadas para la estimación de la eficiencia siguiendo el modelo DEA, junto con las unidades utilizadas para su medición, las fuentes de las que han sido obtenidas y el modo de introducirse en el modelo. Las variables y subíndices utilizados para el cálculo del índice de liberalización se recogen en la Tabla 3. La Tabla 4 presenta los valores medios de las variables usadas en DEA para el periodo considerado.

Las restricciones existentes respecto a los datos del indice de liberalización ferroviaria condicionan la delimitación geográfica y temporal de nuestro ejerci-

\footnotetext{
${ }^{8}$ Este aspecto es especialmente claro en el transporte de pasajeros en el que la propia población se incorpora en el proceso de prestación del servicio de transporte. Por otra parte una población acumulada en un solo punto del territorio anularía cualquier servicio de transporte. Siguiendo el trabajo de Dios, et al. (2006) esta variable podría ser considerada un input fijo en la medida en que viene determinado de forma exógena, dado que está fuera del control de la unidad de decisión, en este caso, el sistema ferroviario.

${ }^{9}$ De los 25 estudios analizados, todos utilizan la mano de obra como input y sólo uno la densidad de población. Entre los outputs prevalecen los estudios que emplean como unidades los pasajeros-kilómetros y las toneladas-kilómetro.
} 
cio. En el ámbito espacial se circunscribe a los países que en cada momento en que se publica el estudio forman parte de la UE, con el añadido de Suiza y Noruega $^{10}$. La vertiente temporal ha de quedar restringida a cuatro años: 2002 , 2004, 2007 y 2011, dado que solamente para estos años se elabora el índice de liberalización.

Tabla 2

Variables utilizadas en el modelo DEA

\begin{tabular}{|c|c|c|c|}
\hline Variable & Unidad & Fuente & Modo \\
\hline $\begin{array}{c}\text { [DEN]: Densidad de población del país titular del } \\
\text { sistema ferroviario. }\end{array}$ & $\begin{array}{c}\text { Habitantes por kilómetro } \\
\text { cuadrado (hab / km }{ }^{2} \text { ) }\end{array}$ & UIC & Input \\
\hline $\begin{array}{c}\text { [LON]: Longitud de las líneas operativas en el } \\
\text { sistema ferroviario a final de cada año. }\end{array}$ & Kilómetros & UIC & Input \\
\hline $\begin{array}{c}\text { [LOC]: Stock de locomotoras y locotractores } \\
\text { (dresinas) operativos a final de cada año. }\end{array}$ & Unidades & UIC & Input \\
\hline $\begin{array}{c}\text { [PER]: Media anual de la fuerza de trabajo en las } \\
\text { empresas que componen el sistema } \\
\text { ferroviario }\end{array}$ & Miles de trabajadores & UIC & Input \\
\hline $\begin{array}{c}\text { [PKM]: Pasajeros transportados por kilómetro en } \\
\text { servicios de tráfico ferroviario comercial }\end{array}$ & $\begin{array}{c}\text { Millones de pasajeros } \\
\text { transportados por kilómetro }\end{array}$ & UIC & Output \\
\hline $\begin{array}{c}\text { [TKM]: Toneladas de mercancías transportadas } \\
\text { por kilómetro en servicios de tráfico } \\
\text { ferroviario comercial }\end{array}$ & $\begin{array}{c}\text { Millones de toneladas de } \\
\text { mercancías transportados por } \\
\text { kilómetro }\end{array}$ & UIC & Output \\
\hline
\end{tabular}

Fuente: Elaboración propia.

Tabla 3

Cálculo del Índice de Liberalización

\begin{tabular}{|c|c|c|}
\hline Índice & Sistemas avanzados & Sistemas con retraso. \\
\hline $\begin{array}{ll}\text { [LIB]: } & \text { cuantificación del grado de } \\
& \text { liberalización del sistema ferroviario } \\
& \text { que opera en el país. Índice de } \\
& \text { liberalización del sistema ferroviario }\end{array}$ & $\begin{array}{l}\text { Valores del índice > : } \\
800 \text { puntos (2007 y 2011) } \\
600 \text { puntos (2002 y 2004) }\end{array}$ & $\begin{array}{l}\text { Valores del índice < : } \\
600 \text { puntos }(2007 \text { y 2011) } \\
300 \text { puntos (2002 y 2004) }\end{array}$ \\
\hline Subíndice & Ponderación & Variables principales \\
\hline $\begin{array}{l}\text { [LEX]: } \\
\text { cuantificación del grado de } \\
\text { liberalización plasmado en la } \\
\text { regulación aplicable }\end{array}$ & $\begin{array}{c}20 \% \\
(45 \%-30 \%-25 \%)\end{array}$ & $\begin{array}{l}\text { - Regulación acceso al mercado } \\
\text { - Autoridad reguladora } \\
\text { - Estructura organizativa } \\
\text { operador heredero del } \\
\text { monopolio público }\end{array}$ \\
\hline $\begin{array}{c}\text { [ACE]: mide las condiciones de apertura } \\
\text { que se presentan en la práctica }\end{array}$ & $\begin{array}{c}80 \% \\
(50 \%-25 \%-20 \%-5 \%)\end{array}$ & $\begin{array}{l}\text { - Barreras operativas } \\
\text { - Accesibilidad mercado } \\
\text { - Barreras administrativas } \\
\text { - Barreras informativas }\end{array}$ \\
\hline
\end{tabular}

Fuente: Elaboración propia a partir del cálculo de los índices de liberalización contenido en Kirchner $(2002,2004,2007$ y 2011).

${ }^{10}$ La Directiva 2001/13 tiene por objeto extender las disposiciones comunitarias a todas las empresas ferroviarias establecidas en la UE para armonizar las condiciones en las que ejercen su actividad e impedir que las licencias constituyan una barrera de entrada al mercado. Esta directiva determina que una licencia obtenida en cualquiera de los países de la UE, Islandia, Liechtenstein, Noruega y Suiza es válida en todo el territorio de la Unión Europea. 
Tabla 4

Valores medios de las variables usadas en DEA en los años de análisis

\begin{tabular}{|c|c|c|c|c|c|c|}
\hline $\begin{array}{c}\text { Sistema } \\
\text { ferroviario }\end{array}$ & $\begin{array}{c}\text { DEN } \\
\left(\mathbf{h a b} / \mathbf{k m}^{2}\right)\end{array}$ & $\begin{array}{l}\text { LON } \\
(\mathbf{k m})\end{array}$ & $\begin{array}{c}\text { LOC } \\
\text { (unidades) }\end{array}$ & $\begin{array}{c}\text { PER } \\
\text { (miles } \\
\text { personas) }\end{array}$ & $\begin{array}{c}\text { PKM } \\
\text { (miles pas } \\
\text { / km) }\end{array}$ & $\begin{array}{c}\text { TKM } \\
\text { (miles } \\
\text { ton / km) }\end{array}$ \\
\hline Alemania & 230,5 & $34.374,5$ & $5.071,4$ & 234 & $74.275,9$ & $89.596,8$ \\
\hline Austria & 98 & 5647,5 & 1214,4 & 45,6 & 9267 & 18904,3 \\
\hline Bélgica & 339,2 & 3529,8 & 693,3 & 38,1 & 9612,7 & 7574 \\
\hline Bulgaria & 68,1 & 4159,4 & 579 & 32,6 & 2362,5 & 4492,7 \\
\hline Chequia & 130 & 9497,1 & 2008,8 & 609,2 & 6638 & 15266,2 \\
\hline Dinamarca & 127 & 2134,3 & 631 & 19,3 & 6377,3 & 2355,5 \\
\hline Eslovenia & 100 & 1226,4 & 160,3 & 8,2 & 793,2 & 3284,6 \\
\hline Eslovaquia & 110,4 & 3635 & 921,1 & 35,6 & 2296,3 & 8898,3 \\
\hline España & 87,5 & 14771,1 & 701,6 & 33,3 & 21665,8 & 10966,9 \\
\hline Estonia & 29,2 & 893,1 & 114,4 & 2,9 & 233 & 8055,4 \\
\hline Finlandia & 15,5 & 5867,9 & 536 & 10,3 & 3662,6 & 9981 \\
\hline Francia & 97,3 & 30809,6 & 4279 & 165,3 & 81011,4 & 38254,3 \\
\hline Grecia & 83 & 2509 & 165,3 & 6,7 & 1725,1 & 588 \\
\hline Holanda & 444 & 2860,8 & 135,2 & 19,7 & 15084,4 & 4889,3 \\
\hline Hungría & 108,7 & 7924,8 & 829,9 & 43,1 & 6547,7 & 7966,7 \\
\hline Irlanda & 60,1 & 1919 & 82,8 & 5,1 & 1753,2 & 220 \\
\hline Italia & 196 & 16795,8 & 3158,9 & 95,8 & 46011,9 & 18807,8 \\
\hline Letonia & 34,9 & 2173,77 & 207,6 & 13,5 & 846,6 & 17015,5 \\
\hline Lituania & 52,6 & 1770,6 & 251,9 & 11,2 & 415,7 & 12783,2 \\
\hline Luxemburgo & 160,1 & 274,9 & 102,8 & 3,2 & 305,4 & 366,7 \\
\hline Noruega & 14,4 & 4094,4 & 69,5 & 6,3 & 2528,8 & 1959,7 \\
\hline Polonia & 122,3 & 19696,4 & 3501,2 & 123,5 & 17222,9 & 41465,5 \\
\hline Portugal & 114,6 & 2554,1 & 180,6 & 8,4 & 3626,7 & 2352 \\
\hline Reino Unido & 249,6 & 18335,2 & 432,2 & 49 & 48531,1 & 20095,8 \\
\hline Rumania & 91,5 & 10889,4 & 1987,9 & 66,4 & 7277,2 & 13279,3 \\
\hline Suecia & 20,1 & 9902,9 & 418,2 & 11,4 & 6330,4 & 10800,8 \\
\hline Suiza & 182,9 & 3416,9 & 1618,4 & 29,2 & 15693 & 11654,8 \\
\hline
\end{tabular}

Fuente: Elaboración propia a partir de UIC.

\section{RESULTADOS}

Los resultados obtenidos se presentan en dos planos, tal como se planteó en el apartado de objetivos. En primer lugar, se muestran los indicadores de eficiencia técnica obtenidos por cada sistema ferroviario, de forma individual, con relación al resto de sistemas y la posible asociación de estos indicadores con los niveles de liberalización desarrollados. En un segundo momento, se realiza una comparación agregada comparando los escenarios creados "ad hoc" con las agrupaciones de países más liberalizados y menos liberalizados. 


\subsection{Resultados individualizados por sistema ferroviario para la eficiencia DEA en una etapa y la eficiencia por programas}

La Tabla 5 recoge los resultados de los indicadores de eficiencia técnica multiservicio estimados por el método DEA y los índices de liberalización de los servicios conjuntos de transportes (pasajeros y mercancías) para los años 2002, 2004, 2007 y 2011, para los 17 únicos sistemas ferroviarios para los que existen datos del índice de liberalización de Kirchner en todos los años considerados. ${ }^{11}$. Para el caso del cálculo de la eficiencia por programas se obtienen los resultados que se plasman en la Tabla 6 . En este caso, dado que se trata de conseguir en una primera etapa las dos muestras de sistemas ferroviarios (más y menos liberalizados) más amplias posibles se opta por introducir en el análisis todos los sistemas para los que se han calculado índices de liberalización, lo que implica asumir la restricción del ámbito temporal a los años 2004, 2007 y 2011.

\section{Tabla 5}

Comparación de eficiencia y liberalización entre sistemas. DEA una etapa

\begin{tabular}{|l|c|c|c|c|c|c|c|c|}
\hline \multirow{2}{*}{$\begin{array}{c}\text { Sistema } \\
\text { ferroviario }\end{array}$} & \multicolumn{2}{c|}{$\mathbf{2 0 0 2}$} & \multicolumn{2}{c|}{2004} & \multicolumn{2}{c|}{2007} & \multicolumn{2}{c|}{2011} \\
\cline { 2 - 9 } & EFIC & LIBER & EFIC & LIBER & EFIC & LIBER & EFIC & LIBER \\
\hline Alemania & 1,0000 & 752 & 1,0000 & 728 & 1,0000 & 826,6 & 1,0000 & 842,2 \\
\hline Austria & 1,0000 & 432 & 1,0000 & 579 & 1,0000 & 788,6 & 1,0000 & 806,2 \\
\hline Bélgica & 0,8342 & 392 & 0,9025 & 461 & 0,7771 & 648,8 & 0,6008 & 753,6 \\
\hline Dinamarca & 1,0000 & 712 & 0,9565 & 693 & 0,9493 & 788,2 & 1,0000 & 825,2 \\
\hline España & 1,0000 & 196 & 0,9131 & 148 & 0,9207 & 630,2 & 0,8266 & 583,4 \\
\hline Finlandia & 1,0000 & 414 & 1,0000 & 542 & 1,0000 & 636 & 1,0000 & 671,4 \\
\hline Francia & 1,0000 & 336 & 1,0000 & 305 & 1,0000 & 573,4 & 1,0000 & 611,6 \\
\hline Grecia & 0,2855 & 204 & 0,2323 & 162 & 0,2222 & 559 & 0,3376 & 571,8 \\
\hline Holanda & 1,0000 & 716 & 1,0000 & 695 & 1,0000 & 809 & 1,0000 & 816,6 \\
\hline Irlanda & 0,3546 & 316 & 0,3116 & 149 & 0,3217 & 332,8 & 0,3364 & 467,6 \\
\hline Italia & 1,0000 & 556 & 1,0000 & 688 & 0,9162 & 675,8 & 0,6914 & 736,6 \\
\hline Luxemburgo & 0,6571 & 272 & 0,6236 & 467 & 0,2515 & 580,6 & 0,2582 & 585 \\
\hline Noruega & 1,0000 & 384 & 1,0000 & 589 & 1,0000 & 698,6 & 1,0000 & 729 \\
\hline Portugal & 0,6458 & 376 & 0,6350 & 668 & 0,6357 & 706,6 & 0,4682 & 737,6 \\
\hline Reino Unido & 1,0000 & 804 & 1,0000 & 781 & 1,0000 & 826,6 & 1,0000 & 865,6 \\
\hline Suecia & 1,0000 & 756 & 1,0000 & 729 & 1,0000 & 825 & 1,0000 & 872 \\
\hline Suiza & 1,0000 & 644 & 1,0000 & 677 & 1,0000 & 756,4 & 1,0000 & 740,4 \\
\hline
\end{tabular}

Fuente: Elaboración propia.

Los indicadores de eficiencia estimados miden distancias respecto a la frontera conformada por unidades eficientes en su combinación de inputs-outputs. Un valor de uno $(1,0)$ indica que los sistemas ferroviarios son eficientes. Cuanto

${ }^{11}$ El resto de sistemas ferroviarios para los que se presentan datos en la Tabla 4 hasta alcanzar los 27 tienen entrada en el modelo únicamente a través del análisis agregado y la inclusión de escenarios que se desarrolla en el apartado 5.3. 
menor es el indicador mayor es el valor de la ineficiencia observada de un sistema ferroviario concreto respecto a los sistemas eficientes.

Para el índice de liberalización, el valor máximo anual que puede alcanzar cada sistema es de 1.000 puntos.

Algunos países muestran sistemas ferroviarios con indicadores de eficiencia completa respecto a la frontera a lo largo de los diversos años considerados: Reino Unido, Holanda, Austria, Finlandia, Alemania, Francia; Noruega, Suecia y Suiza. Empleando como criterio de jerarquización entre los sistemas plenamente eficientes, las veces que el sistema ferroviario del país en cuestión ha sido utilizado en el análisis DEA para formar parte del grupo de referencia o Peer Group resultan como sistemas ferroviarios más eficientes Holanda, con treinta y seis (36) apariciones y Gran Bretaña con veinticinco (25). Este resultado de jerarquización entre los sistemas eficientes se ve confirmado aplicando la técnica de los índices de supereficiencia desarrollada por Andersen y Petersen $(1993)^{12}$. Atendiendo a los resultados de supereficiencia Gran Bretaña presenta claramente el mayor índice para los años 2002 y 2004, mientras que Holanda presenta el tercer mayor índice para los años 2002 y 2007.

Los resultados obtenidos son acordes con otras estimaciones de la literatura sobre eficiencia del sector ferroviario. Destacan los trabajos de Coelli y Perelman (1999) para quienes los países más eficientes son Reino Unido y Holanda; Christopoulos. Loizides y Tsionas (2000) cuyos resultados dan como más eficientes a Francia, Reino Unido, Holanda y Alemania; Coelli y Perelman (2001) de cuyos resultados resulta Holanda como más eficiente; Loizides y Tsionas (2002) que concluyen en su trabajo que el índice de cambio técnico sólo es mayor que la unidad para Alemania y Reino Unido y Asmild, et al. (2009) para quienes la mayor eficiencia recae Reino Unido seguido por Suiza y Holanda.

Los países que presentan mayores índices de ineficiencia en los diversos años son recurrentemente Grecia e Irlanda. Resultados en línea con ello han sido recogidos en la literatura: Hilmola (2006); Coelli y Perelman (1999), Jorge-Moreno y García Cebrián (1999), Christopoulos, Loizides y Tsionas (2000).

Desde una perspectiva temporal la evolución de estos resultados de eficiencia no presenta un patrón claro. Algunos países (ej Alemania) presentan un crecimiento continuado de estos indicadores de eficiencia ${ }^{13}$, pero para un grupo

${ }^{12}$ Andersen y Petersen (1993) proponen un método de clasificación de las unidades eficientes según la metodología DEA (a las que todas las correspondería un índice de eficiencia de 1) consistente en "comparar la unidad que está siendo evaluada con una combinación lineal de todas las demás unidades de la muestra". Por motivos de extensión en el presente trabajo sólo es posible comentar los resultados más destacados.

${ }^{13}$ Alemania presenta unos indicadores de supereficiencia de 107,17 (año 2002), 119,12 (2004), 140,39 (2007) y 180,27 (2011). 
amplio de países (entre otros Bélgica, España, Italia) estas mejoras parecen quebrarse en los ejercicios 2007 y 2011, lo que puede estar reflejando el efecto de la crisis económica sobre el funcionamiento de los sistemas ferroviarios.

Comparando los indicadores de eficiencia y niveles de liberalización obtenidos se observa que los sistemas ferroviarios que aparecen avanzados en el proceso de liberalización respecto la normativa europea, en cada uno de los años del análisis (Reino Unido, Alemania, Suecia y Holanda) presentan elevados indicadores de eficiencia.

Entre los sistemas que presentan eficiencia para todos los años analizados figuran Reino Unido, el sistema ferroviario más liberalizado en los años 2002, 2004 y 2007, Suecia, el sistema con mayor índice de liberalización en 2011, Alemania que empata su puntuación de liberalización con Reino Unido en el año 2007, Holanda, que se sitúa entre los sistemas ferroviarios más avanzados en el proceso de liberalización y, Austria, que ha experimentado un incremento en su grado de liberalización muy elevado en términos relativos durante los últimos años. Estos cinco sistemas ferroviarios presentan un índice de liberalización superior a los 800 puntos en el último año estudiado.

Sin embargo, se observa también que no todos los países que muestran niveles absolutos o muy elevados de eficiencia, presentan un elevado grado de liberalización. Así podemos observar el caso de Finlandia, Francia y Suiza donde la liberalización muestra valores más modestos que no hacen incompatible excelentes resultados en términos de eficiencia.

Por otro lado, se observa que los sistemas ferroviarios con menor grado de liberalización, normalmente, se encuentran entre los relativamente más ineficientes. Grecia e Irlanda son los países donde más claramente se muestra esta asociación. Sin embargo, rompe este axioma el caso de España, que aunque presenta valores muy bajos del índice de liberalización para los años 2002 y 2004, principalmente debido a la falta de independencia entre operación y gestión de infraestructura, sus resultados de ineficiencia no son de los más acusados, alcanzando incluso la eficiencia en el año 2002.

Otro resultado de interés es el observado para un cierto grupo de sistemas ferroviarios en los que se han producido procesos paralelos de incrementos del grado de liberalización unidos a decrementos en la eficiencia técnica estimada. Entre ellos destacan España, Italia o Portugal. A nivel temporal se constatan dos efectos: por un lado, el grado de liberalización experimenta un incremento a una tasa decreciente conforme avanza el tiempo; por otro, el grado de eficiencia técnica no mejora con el paso de los años, retrocede para cada uno de los años considerados a una tasa aproximada de entre el $3 \%$ y el $8 \%$.

En definitiva, desde la perspectiva de un análisis individualizado por países, parecen existir dificultades para obtener resultados concluyentes. Aunque los sistemas muy liberalizados obtienen buenos resultados de eficiencia, no todos 
los países eficientes precisan de la liberalización. Tampoco está suficientemente clara la asociación en los sistemas con escaso grado de liberalización, pues no todos presentan amplias ineficiencias. Por último, existe un grupo de sistemas ferroviarios en los que se observa una caída del nivel de eficiencia conforme han ido avanzando en sus niveles de liberalización. Dada la falta de resultados concluyentes es necesario acudir a un contraste estadístico que permita determinar la existencia de relación entre ambas variables a nivel global.

Tabla 6

Comparación de eficiencia y liberalización entre sistemas. DEA por programas

\begin{tabular}{|c|c|c|c|c|c|c|}
\hline \multirow{2}{*}{$\begin{array}{c}\text { Sistema } \\
\text { ferroviario }\end{array}$} & \multicolumn{2}{|c|}{2004} & \multicolumn{2}{|c|}{2007} & \multicolumn{2}{|c|}{2011} \\
\hline & EFIC & LIBER & EFIC & LIBER & EFIC & LIBER \\
\hline Alemania & 0,9227 & 728 & 0,9901 & 826,6 & 1,0000 & 842,2 \\
\hline Austria & 0,7558 & 579 & 0,7364 & 788,6 & 0,8049 & 806,2 \\
\hline Bélgica & 0,6714 & 461 & 0,6540 & 648,8 & 0,5995 & 753,6 \\
\hline Bulgaria & - & - & 0,2928 & 652,4 & 0,2134 & 718,2 \\
\hline Dinamarca & 0,7278 & 693 & 0,6743 & 788,2 & 1,0000 & 825,2 \\
\hline Eslovaquia & 0,4044 & 458 & 0,3749 & 700,2 & 0,3223 & 737,8 \\
\hline Eslovenia & 0,4013 & 326 & 0,4205 & 664,4 & 0,4116 & 671,8 \\
\hline España & 0,8385 & 148 & 1,0000 & 630,2 & 0,9504 & 583,4 \\
\hline Estonia & 1,0000 & 257 & 1,0000 & 691,6 & 1,0000 & 729,6 \\
\hline Finlandia & 0,9440 & 542 & 1,0000 & 636 & 1,0000 & 671,4 \\
\hline Francia & 1,0000 & 305 & 1,0000 & 573,4 & 1,0000 & 611,6 \\
\hline Grecia & 0,2630 & 162 & 0,2387 & 559 & 0,5900 & 571,8 \\
\hline Holanda & 1,0000 & 695 & 1,0000 & 809 & 1,0000 & 816,6 \\
\hline Hungría & 0,4161 & 366 & 0,3354 & 636,6 & 0,2249 & 657,2 \\
\hline Irlanda & 0,3830 & 149 & 0,3525 & 332,8 & 0,6165 & 467,6 \\
\hline Italia & 1,0000 & 688 & 0,9471 & 675,8 & 0,7766 & 736,6 \\
\hline Letonia & 1,0000 & 516 & 1,0000 & 650,2 & 1,0000 & 587,2 \\
\hline Lituania & 0,7105 & 222 & 0,9663 & 684 & 0,9660 & 592,4 \\
\hline Luxemburgo & 0,3620 & 467 & 0,2557 & 580,6 & 0,2528 & 585 \\
\hline Noruega & 1,0000 & 589 & 0,9587 & 698,6 & 0,7761 & 729 \\
\hline Polonia & 0,8732 & 549 & 0,8526 & 739 & 0,6764 & 736,6 \\
\hline Portugal & 0,4446 & 668 & 0,4103 & 706,6 & 0,5455 & 737,6 \\
\hline Reino Unido & 1,0000 & 781 & 1,0000 & 826,6 & 1,0000 & 865,6 \\
\hline Rep. Checa & 0,4002 & 548 & 0,3959 & 738,2 & 0,3810 & 738 \\
\hline Rumanía & - & - & 0,3767 & 722 & 0,3126 & 725,4 \\
\hline Suecia & 1,0000 & 729 & 1,0000 & 825 & 1,0000 & 872 \\
\hline Suiza & 1,0000 & 677 & 1,0000 & 756,4 & 1,0000 & 740,4 \\
\hline
\end{tabular}

Fuente: Elaboración propia.

De los resultados obtenidos en el análisis de la eficiencia por programas se desprende en primer lugar como el cálculo de la eficiencia debida a la variable de entorno (liberalización) se muestra más exigente respecto a los 17 sistemas a los que se aplica la técnica DEA en una etapa. De ellos, solamente se mantienen como eficientes en todos los años considerados los sistemas de Francia, Ho- 
landa, Reino Unido, Suecia y Suiza. Se incorporan a este grupo de máxima eficiencia los sistemas de Estonia y Letonia, situados en la parte baja del índice de liberalización. Esta incorporación está en coherencia con los resultados de Hilmola (2006), donde precisamente estos dos sistemas se muestran como los más eficientes. Entre los países menos eficientes se mantienen Grecia, Irlanda y Luxemburgo, a los que se suman Bulgaria y Hungría.

Por otra parte aumenta el grupo de sistemas que presentan procesos temporales paralelos de descensos de índices de liberalización junto con incrementos de eficiencia, en concreto entre los nuevos sistemas introducidos en el análisis, Bulgaria, Eslovaquia, Hungría, y Rumanía experimentan estos procesos.

\subsection{Contraste a nivel global. Estadístico Kruskal-Wallis}

En nuestro caso, mediante la aplicación del contraste Kruskal-Wallis se quiere contrastar si los dos grupos de sistemas ferroviarios identificados, sistemas ferroviarios eficientes e ineficientes son iguales o diferentes en su grado de liberalización. Dado que por definición la técnica DEA no discrimina entre sistemas eficientes, asigna a todos un valor de uno, se requiere aplicar un contraste por diferencia de medias como el que proporciona el estadístico KruskalWallis. Esta metodología permite introducir la variable liberalización en su forma cuantificada, una de las aportaciones de este trabajo y no como se hace habitualmente en la literatura como variable dicotómica. La aplicación de este estadístico frente a las metodologías de dos etapas, como el DEA por programas que incluyesen la variable liberalización como variable de entorno, se encuentra en que es posible realizar comparaciones cuantitativas de los grados de liberalización entre los sistemas ferroviarios y lo que es más importante no exige a priori decantarse por la inclusión de los sistemas en la categoría liberalizados o no liberalizados, cuando como se ha expuesto en el sector ferroviario el grueso de los sistemas se encuentran en grados de liberalización intermedios y son muy pocos los sistemas completamente liberalizados (Reino Unido, Suecia y en menor medida Alemania) o nada liberalizados (Irlanda y en menor medida Grecia).

Para proceder al cálculo del estadístico se genera una variable dummy que toma el valor 1 cuando el sistema ferroviario es eficiente y el valor 0 cuando es ineficiente, según los resultados del análisis DEA. Queda conformada así la "variable de agrupación" que va a permitir contrastar si los valores que presentan en la variable liberalización los dos grupos de sistemas ferroviarios son estadísticamente iguales en media/mediana o no lo son.

A continuación se presenta la Tabla 7 con el cálculo de los estadísticos de contraste junto con sus p-valores asociados, correspondientes a los cuatro años estudiados para cada uno de los escenarios y años diferentes. 
Tabla 7

Contraste Kruskal-Wallis aplicado a DEA en una etapa por años

\begin{tabular}{|c|c|c|c|c|}
\hline AÑO & $\mathbf{2 0 0 2}$ & $\mathbf{2 0 0 4}$ & $\mathbf{2 0 0 7}$ & $\mathbf{2 0 1 1}$ \\
\hline Estadístico & 6,259 & 3,438 & 5,044 & 8,571 \\
(p-valor) & $(0,012)$ & $(0,064)$ & $(0,025)$ & $(0,003)$ \\
\hline
\end{tabular}

Fuente: Elaboración propia.

A la vista de los resultados obtenidos puede decirse que las variables contrastadas, liberalización y eficiencia están asociadas positivamente, si bien no de forma absoluta. En la población de la que provienen las muestras estudiadas, están relacionadas las diferencias apreciadas en los niveles de liberalización de los diferentes grupos de sistemas ferroviarios con motivos de eficiencia o ineficiencia del sistema. Existe por tanto una diferencia intrínseca general entre los grupos de sistemas ferroviarios eficientes e ineficientes comparados en cuanto a niveles de liberalización se refiere. En relación con los resultados obtenidos para los sistemas de forma individualizada, supone que la ponderación de los sistemas en los que hay relación entre las variables estudiadas (principalmente los que ocupan las primeras y últimas posiciones en el ranking del grado de liberalización) supera al peso que tienen los sistemas que se presentan como excepciones a esta regla.

Para estudiar diferencias debidas a la variable de entorno (liberalización) en la eficiencia en dos etapas, en la Tabla 8 se recogen los resultados del contraste de diferencia de medias Kruskal-Wallis aplicado sobre las holguras provenientes de la segunda etapa de la metodología de eficiencia por programas, tal y como proponen Charnes, et al. (1981).

Tabla 8

Contraste Kruskal-Wallis aplicado a DEA por programas por holguras de las variables

\begin{tabular}{|c|c|c|c|c|}
\hline VARIABLE-Slack & Long-S & Dens-S & Loco-S & Pers-S \\
\hline Estadístico & 0,000 & 1,000 & 7,856 & 5,302 \\
(p-valor) & $(1,000)$ & $(0,317)$ & $(0,005)$ & $(0,021)$ \\
\hline
\end{tabular}

Fuente: Elaboración propia.

El análisis de estos resultados lleva a la conclusión que la variable liberalización está relacionada con la eficiencia debida a las variables número de locomotoras operativas y personal anual medio. Por tanto, en los sistemas donde se han implementado las políticas de liberalización en mayor grado han venido acompañadas de mejoras de eficiencia, gracias precisamente a aquellas variables sobre las que los sistemas pueden intervenir, los medios de producción de material circulante y mano de obra. Dada la naturaleza de coste hundido inherente a la variable kilómetros operativos de vía, la actuación sobre la misma es muy limitada en el corto plazo. Tampoco parece factible incidir en la variable 
densidad de población con fines únicamente de política de transporte. En cualquier caso los resultados relativos a estas dos últimas variables deben ser tomados con cautela dado que son los que menos casos de holgura entre los sistemas presentan.

\subsection{Resultados del análisis agregado. Inclusión de escenarios}

En este punto se plantean los resultados derivados de la introducción de dos escenarios en el análisis, el que recoge los veinte (20) sistemas ferroviarios más liberalizados cada año (Escenario A) y el formado con los veinte (20) sistemas con un menor índice de liberalización cada año (Escenario B). Por tanto la conformación de estos escenarios implica, respecto al análisis anterior, que los sistemas incorporados cada año en cada escenario son variables, en función de aquellos sistemas ferroviarios para los que el índice de liberalización de Kirchner presenta un mayor (escenario A) o menor (escenario B) valor cada año. La conformación de cada escenario con un número de 20 obedece a las recomendaciones de la literatura del análisis DEA para la comparabilidad óptima de unidades. $^{14}$

La Tabla 9 muestra, para cada uno de los años objeto de estudio, las ineficiencias medias que presenta cada uno de los escenarios y, entre paréntesis, el número de sistemas ineficientes en cada uno de ellos.

Tabla 9

Resultados de ineficiencia media por escenario

\begin{tabular}{|l|c|c|c|c|c|}
\hline & $\begin{array}{c}\text { AÑO 2002 } \\
\text { (inicial) }\end{array}$ & AÑO 2004 & AÑO 2007 & AÑO 2011 & TOTAL \\
\hline $\begin{array}{l}\text { ESCENARIO A } \\
\text { (+liberalizados) }\end{array}$ & $0,5096677(3)$ & $0,21274(6)$ & $0,290564(7)$ & $0,286033(10)$ & $0,296144(26)$ \\
\hline $\begin{array}{l}\text { ESCENARIO B } \\
\text { (- liberalizados) }\end{array}$ & $0,5096677(3)$ & $0,38450(7)$ & $0,332893(8)$ & $0,2972888(9)$ & $0,354048(27)$ \\
\hline TOTAL & $0,5096677(6)$ & $0,3052(13)$ & $0,3131(15)$ & $0,291364(19)$ & $0,3256230(53)$ \\
\hline
\end{tabular}

Fuente: Elaboración propia.

La Tabla 10 presenta los datos medios del índice de liberalización estimados para los diferentes escenarios y años considerados a los efectos de poder comparar con los resultados de ineficiencia.

\footnotetext{
${ }^{14}$ Banker, et al. (1989) establecían, a modo orientativo, el requisito de que el número de unidades analizadas sea mayor o igual a la suma de inputs y outputs para que el modelo tenga carácter discriminatorio. Otros autores como Norman y Stoker (1991) mencionan que 20 unidades serían suficientes sin hacer depender el número de la cantidad de variables o Mancebón (1996) recoge la recomendación de que el número de entidades analizadas sea al menos el triple de las variables relevantes introducidas en el modelo. En el presente trabajo adoptando el número de 20 unidades (sistemas ferroviarios) se consiguen satisfacer las tres pautas.
} 
Tabla 10

Resultados de liberalización media por escenario

\begin{tabular}{|l|c|c|c|c|c|}
\hline & $\begin{array}{c}\text { AÑO 2002 } \\
\text { (inicial) }\end{array}$ & AÑO 2004 & AÑO 2007 & AÑO 2011 & TOTAL \\
\hline ESCENARIO A & 486 & 568,25 & 729,61 & 755,5 & 640,63 \\
\hline ESCENARIO B & 486 & 433,85 & 646,02 & 666,43 & 560,88 \\
\hline TOTAL & 486 & 501,05 & 687,81 & 734,69 & 606,92 \\
\hline
\end{tabular}

Fuente: Elaboración propia.

De un análisis comparativo se evidencia como el escenario que presenta el mayor índice de liberalización (escenario A), es el que resulta con un menor grado de ineficiencia media a lo largo del periodo $(0,2961)$ y para cada uno de los años analizados. Mientras que el escenario que contiene los sistemas menos liberalizados y presenta menor índice de liberalización (escenario B), es precisamente el que muestra un mayor grado de ineficiencia (0,35404). Se cumple por tanto una asociación de tipo ordinal de tal manera que el escenario que contiene los sistemas ferroviarios más (menos) liberalizados es también el que presenta un nivel de ineficiencia menor (mayor).

Atendiendo a un criterio temporal la evolución de los índices agregados muestra como el grado de liberalización aumenta a una tasa creciente hasta 2007, año en el que sigue creciendo, pero desacelera el ritmo de crecimiento. Dado que no se produce una caída paralela de la ineficiencia con el paso del tiempo, no es posible establecer una asociación temporal entre la evolución de ambas variables, el crecimiento del índice de liberalización no va acompañado en el tiempo de una paralela reducción de la ineficiencia en todos los escenarios. Esta asociación sólo se produce en el escenario B en el que los tímidos avances en el grado de liberalización de los sistemas que conforman el escenario van acompañados de reducciones en los niveles de ineficiencia técnica, hasta 0,2972 en 2011.

Desde la perspectiva de la evolución temporal se advierte que los resultados de ineficiencia media disminuyen con el paso del tiempo solamente para el caso del escenario B. De alguna forma la ganancia marginal de mayor liberalización es cada vez menor para los sistemas que están ya muy liberalizados pero todavía es positiva para aquellos que están menos liberalizados. En estos sistemas existiría margen para que medidas liberalizadoras generasen ganancias en términos de eficiencia.

\section{CONCLUSIONES}

Este trabajo analiza la existencia de evidencia empírica sobre la asociación entre el grado de liberalización desarrollado por los principales sistemas ferroviarios europeos y el nivel de eficiencia técnica alcanzado por dichos sistemas. Las principales conclusiones encontradas son las siguientes: 
1) Los resultados obtenidos muestran que la asociación entre las variables liberalización y eficiencia técnica funciona bien para los sistemas ferroviarios más liberalizados, de tal forma que aquellos sistemas con mayores índices de liberalización presentan óptimos resultados de eficiencia, como sucede claramente con Reino Unido, Suecia, Alemania, Holanda y en cierta medida con Austria.

2) No obstante, también se observa que esta relación no es bidireccional y no todos los sistemas ferroviarios que alcanzan altos niveles de eficiencia se caracterizan por tener un elevado índice de liberalización (Suiza, Letonia, Estonia o Finlandia). Ello puede evidenciar que la liberalización puede postularse, sin duda, como un factor que puede contribuir a la eficiencia, pero que no es en absoluto el único. El logro de la misma depende también de otros factores lo que permite que pueda ser alcanzada igualmente en contextos menos liberalizados. La eficiencia podría explicarse por otros factores como la competitividad del ferrocarril frente a otros modos, el impulso de políticas de transporte que incentiven el uso del modo ferroviario o la tecnología aplicable y no sólo por el grado de liberalización del sector.

3) La posible asociación entre liberalización y eficiencia tampoco es concluyente para los países que presentan bajos índices de liberalización. Aunque para algunos países (Grecia e Irlanda) sí se cumple esta asociación, en otros, como el caso de España, bajos niveles de liberalización no han provocado necesariamente ineficiencias acusadas.

4) Asimismo, otro resultado de interés muestra que existe un grupo de sistemas ferroviarios en los que se producen a lo largo del tiempo procesos paralelos de incrementos del grado de liberalización acompañados de descensos en los niveles de eficiencia técnica, lo que impide generalizar la asociación a todos los sistemas ferroviarios estudiados. Este es el caso de Bélgica, Portugal o Italia.

5) Los resultados del contraste Kruskal-Wallis sobre las eficiencias en una etapa inciden en la línea apuntada de la existencia evidente de una asociación positiva en términos globales entre eficiencia productiva y liberalización, si bien no puede considerarse una regla fija extrapolable a todos los años y sistemas estudiados. De la aplicación del estadístico Kruskal-Wallis sobre las holguras provenientes de la segunda etapa, se deduce que los sistemas más liberalizados son precisamente aquellos que muestran más eficiencia en las variables que están bajo su control, material rodante y mano de obra. Estas variables son las que muestran un mayor número de holguras por lo que la fiabilidad del resultado es mayor. Se confirman así los resultados obtenidos en la eficiencia DEA en una etapa de existencia de evidencia estadística suficiente para afirmar la asociación entre las variables estudiadas, lo que permite además confirmar la robustez del modelo. 
6) La comparativa planteada de forma agregada entre escenarios dinámicos que van incorporando a los países con sistemas ferroviarios más liberalizados y menos liberalizados permite observar una clasificación ordinal favorable a la existencia de asociación entre las variables eficiencia y liberalización. De este modo, el escenario que contiene los sistemas ferroviarios más (menos) liberalizados es también el que presenta un nivel de ineficiencia media menor (mayor) para todos los años considerados.

En definitiva, a partir de la evidencia empírica obtenida, es posible establecer globalmente una asociación positiva, si bien de carácter débil, por la que un mayor grado de liberalización va acompañado generalmente por mejoras en la eficiencia del sistema ferroviario. Sin embargo, a partir de un estudio individualizado de los sistemas ferroviarios es posible localizar empíricamente la existencia de excepciones a la asociación entre liberalización y eficiencia. Por tanto, no puede determinarse un modelo de sistema ferroviario con un grado de liberalización óptimo, que pueda ser utilizado de referente, para asegurar el logro de la eficiencia. Ni siquiera es posible fijar un modelo de gestión ferroviaria que garantice obtener los mejores resultados de eficiencia. Modelos de gestión tan dispares como el británico (separación con gestor privado), el holandés y sueco (separación con gestor público) o el alemán y austriaco (integración vertical pública) comparten las cotas más altas de eficiencia técnica que resultan del trabajo.

Tal y como se ha podido comprobar a partir del trabajo desarrollado las principales limitaciones del modelo derivan de la ausencia de datos para el grado de liberalización de los sistemas ferroviarios en forma de una variable continua que presente valores para todos los años considerados. Esta carencia que no acaece con los datos que permiten calcular la variable eficiencia, dado que la UIC publica anualmente estos datos. Las posibles extensiones del modelo podrían partir por un lado del cálculo de la eficiencia dinámica de los sistemas ferroviarios y el análisis de su variación en el tiempo, estos resultados que atienden a la variación de la eficiencia sin embargo no podrían compararse con los del grado liberalización a causa de la limitación comentada. Por otro lado, la inclusión de variables de entorno más allá del grado de liberalización como pueden ser el grado de electrificación de la vía o la proporción de vías de alta velocidad respecto al total, el número de anchos diferentes, el índice de ocupación de los trenes o el grado de utilización de la red (medida como el número medio de trayectos por línea) darían lugar a próximos estudios muy interesantes sobre cómo juega cada una de estas variables sobre la eficiencia del sistema ferroviario en su conjunto. Finalmente, la aplicación de la metodología de cálculo de la eficiencia por programas con 3 etapas, Dios, et al. (2006), permitiría separar el efecto de la variable entorno que se incluyese en el trabajo de la eficiencia de cada sistema ferroviario, mediante el cálculo de índices de eficien- 
cia de cada sistema que ya no contienen el efecto de la variable entorno ni del error.

\section{REFERENCIAS BIBLIOGRÁFICAS}

AFFUSO, L., ANGERIZ, A. y POLLITT, M. (2002). "Measuring the Efficiency of Britain's Privatised Train Operating Companies" en Regulation Initiative Discussion Paper Series, Number 48. London LBS.

ANDERSEN, P. y PETERSEN, N.C. (1993). "A procedure for Ranking Efficient Units in Data Envelopment Analysis" en Management Science, 39 (10): 1261-1264.

ASMILD, M.; HOLVAD, T.; HOUGAARD, J.L. y KRONBORG, D. (2009). "Railway reforms: do they influence operating efficiency" en Transportation. September 2009, Volume 36, Issue 5, pp 617-638".

BANKER, R.D., CHARNES, A., COOPER, W.W., SWARTS, J. y THOMAS, D.A. (1989). "An introduction to data envelopment analysis with some of its models and their uses" en Research in Governmental and Nonprofit Accounting. Vol. 5: 125-163. JAI Press, Greenwich

CANTOS, P. PASTOR, J.M. y SERRANO, L (2000). "Efficiency measures and output specification: The case of European railways" en Journal of Transportation and Statistics, vol. 3 (3), 61-68.

CANTOS, P. PASTOR, J.M. y SERRANO, L (2010). "Vertical and horizontal separation in the European Railway Sector and its effects on Productivity" en Journal of Transport Economics and Policy, vol.44(2), 139-160.

CHARNES, A., W. COOPER y RHODES, E. (1981). "Evaluating Program and Managerial Efficiency: An Application of Data Envelopment Analysis to Program Follow Through", en Management Science, 27(6), 668-697.

CHRISTOPOULOS, D. LOIZIDES, J. y TSIONAS, E. (2000). "Measuring input specific technical inefficiency in European railways. A pannel data approach" en International Journal of Transport Economics Vol. 27.2000, 2, p. 147-171.

COWIE, J. (1999). "The technical efficiency of public and private ownership in the rail industry. The case of Swiss private railways" en Journal of Transport Economics Policy Volume 33, Pages 241-252.

COELLI, T., PRASADA RAO, D.S. y BATTESE, G.E. (1998). An Introduction to Efficiency and Productivity Analysis. Kluwer Academic Publishers, Boston.

COELLI, T. y PERELMAN, S. (1999). "A comparison of parametric and non-parametric distance functions: with aplication of European railways" en European Journal of Operational Research. Volume 117, Issue 2, 1 September 1999, Pages 326-339

COELLI, T. y PERELMAN, S. (2001). "Medición de la eficiencia técnica en contextos multiproducto", en Álvarez Pinilla, A. (coord), La medición de la eficiencia y la productividad, (pp. 113-135), Madrid: Ediciones Pirámide.

DIOS, R., MARTÍNEZ, J.M. y MARTÍNEZ CARRASCO, F. (2006). "El análisis de eficiencia con variables de entorno: un método de programas con tres etapas" en Estudios de Economía Aplicada, vol. 24, núm. 1, abril 2006, pp. 477-497. 
ESTEBAN, J. y COLL, V. (2003). "Competitividad y eficiencia" en Estudios de Economía Aplicada, vol. 23, núm.1, pp. 423-450.

FARRELL M. (1957). "The measurement of productive efficiency" en Journal of the Royal Statistical Society, Series A, General, 120, part 3, pp. 253-290.

FRIEBEL, G., IVALDI, M. y VIBES, C. (2003). "Railway (De) Regulation. A European Efficiency Comparison”. Rail Transport, IDEI Report \# 3, Institut D’Economie Industrielle.

GOLANY, B. y ROLL, Y. (1989). "An Application Procedure for DEA" en Omega. Volume 17, Issue 3, 1989, Pages 237-250.

GUARNIDO, A., JAEN, M. y AMATE, I. (2007). La desregulación y privatización de las empresas públicas. El caso de las telecomunicaciones en España. Universidad de Almería D.L. Volumen 37.

HILMOLA, O.-P. (2006). "European railway freight transportation and adaptation to demand decline: Efficiency and partial productivity analysis from period of 1980-2003" en International Journal of Productivity and Performance Management, Vol. 56 Iss: 3, pp. $205-225$.

DE JORGE-MORENO, J. y GARCIA, L. (1999). "Measuring of production efficiency in European railways" en European Business Review, Vol. 99 Iss: 5, pp.332 - 344

KIRCHNER, C. (2002, 2004, 2007 y 2011). Summary of the study rail liberalisation index. Berlín: Universidad Humbolt.

LOIZIDES, J. y TSIONAS, E.G. (2002). "Productivity growth in European railways:a new approach" en Transportation Research Part A: Policy and Practice. Volume 36, Issue 7, August 2002, Pages 633-644

MANCEBÓN, M. J. (1996). La evaluación de la eficiencia de los centros escolares públicos. Tesis Doctoral, Universidad de Zaragoza

NORMAN, M. y STOKER, B. (1991). Data Envelopment Analysis, The Assessment of Performance. John Wiley, Chichester.

OUM, T. y YU, C. (1994). "Economic efficiency of railways and implications for public policy" en Journal of Transport Economics and Policy, vol. 28, pp. 121-138

PASTOR, J.T. (2000). "Global efficiency measures in DEA". II Oviedo Workshop. May 2000.

WETZEL, H. (2008). "European Railway Deregulation: The Influence of Regulatory and Environmental Conditions on Efficiency" en Working Paper Series in Economics \# 86. University of Lüneburg. 


\section{ANEXO}

En este anexo se presentan y comentan los resultados de la aplicación de la técnica conglomerados jerárquicos con dos (2) variantes. En la primera se emplean las variables input y output relacionadas con el nivel de población (número de habitantes) de cada país donde se asienta el sistema ferroviario estudiado.

Historial de conglomeración 1

\begin{tabular}{|c|c|c|c|c|c|c|}
\hline \multirow[t]{2}{*}{ Etapa } & \multicolumn{2}{|c|}{ Conglomerado que se combina } & \multirow[t]{2}{*}{ Coeficientes } & \multicolumn{2}{|c|}{$\begin{array}{c}\text { Etapa en la que el } \\
\text { conglomerado aparece por } 1^{\mathrm{a}} \\
\text { vez }\end{array}$} & \multirow{2}{*}{$\begin{array}{c}\text { Próxima } \\
\text { etapa }\end{array}$} \\
\hline & $\begin{array}{c}\text { Conglomerado } \\
1\end{array}$ & $\begin{array}{c}\text { Conglomerado } \\
2\end{array}$ & & \begin{tabular}{|c|} 
Conglomerado \\
1
\end{tabular} & $\begin{array}{c}\text { Conglomerado } \\
2\end{array}$ & \\
\hline 1 & 7 & 24 & 84,255 & 0 & 0 & 2 \\
\hline 2 & 7 & 26 & 570,057 & 1 & 0 & 15 \\
\hline 3 & 10 & 12 & 741,152 & 0 & 0 & 11 \\
\hline 4 & 22 & 23 & 755,066 & 0 & 0 & 10 \\
\hline 5 & 19 & 21 & 766,269 & 0 & 0 & 9 \\
\hline 6 & 6 & 15 & 917,052 & 0 & 0 & 14 \\
\hline 7 & 3 & 20 & 941,746 & 0 & 0 & 9 \\
\hline 8 & 5 & 8 & 1147,451 & 0 & 0 & 19 \\
\hline 9 & 3 & 19 & 1909,092 & 7 & 5 & 11 \\
\hline 10 & 18 & 22 & 2419,551 & 0 & 4 & 17 \\
\hline 11 & 3 & 10 & 2509,890 & 9 & 3 & 15 \\
\hline 12 & 1 & 4 & 5707,438 & 0 & 0 & 18 \\
\hline 13 & 13 & 25 & 5881,405 & 0 & 0 & 19 \\
\hline 14 & 6 & 14 & 5919,571 & 6 & 0 & 20 \\
\hline 15 & 3 & 7 & 6076,829 & 11 & 2 & 23 \\
\hline 16 & 11 & 16 & 6489,912 & 0 & 0 & 17 \\
\hline 17 & 11 & 18 & 7928,179 & 16 & 10 & 18 \\
\hline 18 & 1 & 11 & 10752,872 & 12 & 17 & 20 \\
\hline 19 & 5 & 13 & 15283,053 & 8 & 13 & 24 \\
\hline 20 & 1 & 6 & 21457,733 & 18 & 14 & 23 \\
\hline 21 & 2 & 9 & 22760,188 & 0 & 0 & 22 \\
\hline 22 & 2 & 27 & 33495,889 & 21 & 0 & 25 \\
\hline 23 & 1 & 3 & 34283,277 & 20 & 15 & 24 \\
\hline 24 & 1 & 5 & 39094,110 & 23 & 19 & 26 \\
\hline 25 & 2 & 17 & 73039,807 & 22 & 0 & 26 \\
\hline 26 & 1 & 2 & 159621,149 & 24 & 25 & 0 \\
\hline
\end{tabular}

Fuente: Elaboración propia.

Adoptando como solución óptima de conglomerados el número de dos, existiría por un lado un conglomerado 1 con 23 sistemas ferroviarios y otro conglomerado 2 (que denominaremos "centroeuropeo"), con cuatro sistemas: Bélgica, Alemania, Holanda y Suiza. La adopción de tres conglomerados su- 
pone formar un conglomerado unitario, solamente con Holanda, y mantener el conglomerado del grupo principal y el conglomerado "centro europeo" sin Holanda.

En la segunda variante se emplean en el análisis de conglomerados jerárquico las variables input relacionadas con el nivel de población (número de habitantes) y las output medidas en función de los kilómetros de red ferroviaria operativa para cada país donde se asienta el sistema ferroviario estudiado.

Historial de conglomeración 2

\begin{tabular}{|c|c|c|c|c|c|c|}
\hline \multirow[t]{2}{*}{ Etapa } & \multicolumn{2}{|c|}{ Conglomerado que se combina } & \multirow[t]{2}{*}{ Coeficientes } & \multicolumn{2}{|c|}{$\begin{array}{c}\text { Etapa en la que el } \\
\text { conglomerado aparece por } 1^{\mathrm{a}} \\
\text { vez }\end{array}$} & \multirow{2}{*}{$\begin{array}{c}\text { Próxima } \\
\text { etapa }\end{array}$} \\
\hline & \begin{tabular}{|c|} 
Conglomerado \\
1
\end{tabular} & $\begin{array}{c}\text { Conglomerado } \\
2\end{array}$ & & $\begin{array}{c}\text { Conglomerado } \\
1\end{array}$ & $\begin{array}{c}\text { Conglomerado } \\
2\end{array}$ & \\
\hline 1 & 7 & 26 & 35,963 & 0 & 0 & 3 \\
\hline 2 & 8 & 23 & 63,785 & 0 & 0 & 6 \\
\hline 3 & 7 & 24 & 91,554 & 1 & 0 & 12 \\
\hline 4 & 12 & 15 & 114,337 & 0 & 0 & 10 \\
\hline 5 & 10 & 21 & 124,179 & 0 & 0 & 15 \\
\hline 6 & 1 & 8 & 154,413 & 0 & 2 & 11 \\
\hline 7 & 11 & 22 & 167,672 & 0 & 0 & 14 \\
\hline 8 & 5 & 18 & 195,042 & 0 & 0 & 16 \\
\hline 9 & 6 & 14 & 220,399 & 0 & 0 & 12 \\
\hline 10 & 3 & 12 & 282,982 & 0 & 4 & 15 \\
\hline 11 & 1 & 20 & 326,080 & 6 & 0 & 14 \\
\hline 12 & 6 & 7 & 466,090 & 9 & 3 & 20 \\
\hline 13 & 16 & 27 & 654,412 & 0 & 0 & 19 \\
\hline 14 & 1 & 11 & 746,146 & 11 & 7 & 16 \\
\hline 15 & 3 & 10 & 785,235 & 10 & 5 & 20 \\
\hline 16 & 1 & 5 & 889,493 & 14 & 8 & 18 \\
\hline 17 & 9 & 25 & 970,771 & 0 & 0 & 21 \\
\hline 18 & 1 & 19 & 1609,151 & 16 & 0 & 22 \\
\hline 19 & 13 & 16 & 2525,508 & 0 & 13 & 21 \\
\hline 20 & 3 & 6 & 2654,419 & 15 & 12 & 23 \\
\hline 21 & 9 & 13 & 5003,742 & 17 & 19 & 25 \\
\hline 22 & 1 & 4 & 5011,755 & 18 & 0 & 23 \\
\hline 23 & 1 & 3 & 7229,934 & 22 & 20 & 25 \\
\hline 24 & 2 & 17 & 13236,789 & 0 & 0 & 26 \\
\hline 25 & 1 & 9 & 20910,628 & 23 & 21 & 26 \\
\hline 26 & 1 & 2 & 92854,194 & 25 & 24 & 0 \\
\hline
\end{tabular}

Fuente: Elaboración propia.

Para la primera división en dos conglomerados aparece separado del grupo principal un conglomerado de dos países Holanda y Bélgica (conglomerado "Bene"). La siguiente iteración que presenta tres conglomerados separa del conglomerado principal de sistemas, además del comentado conglomerado "Bene", 
un conglomerado formado por los siguientes sistemas ferroviarios: Alemania, Italia, Luxemburgo, Reino Unido y Suiza. En esta segunda iteración, por definición, las similitudes dentro del conglomerado son menos evidentes.

Por tanto como resultado común a ambas variantes se puede concluir que la primera iteración no produce en ninguna de ellas dos grandes conglomerados, lo que implicaría una heterogeneidad estructural dentro del grupo de sistemas estudiado. Los conglomerados formados son pequeños (4 y 2 sistemas respectivamente) y con coeficientes de heterogeneidad bajos en relación con los 27 sistemas estudiados, lo que no justifica su separación del grupo. Tampoco las segundas iteraciones provocan grandes conglomerados separados del grupo principal. A lo que se añade que de conformidad con la metodología empleada según se avanza en el número de conglomerados el grado de homogeneidad dentro del conglomerado disminuye. Queda así contrastado que no existe una heterogeneidad suficiente en el grupo de sistemas ferroviarios estudiados que impida su inclusión conjunta como grupo de referencia en estudios DEA. 